\title{
Zweifelsfragen der gesamtschuldnerischen Organhaftung im Aktienrecht
}

\author{
Zur Bewährung der bürgerlich-rechtlichen Institute der \\ „gestörten Gesamtschuld“ und „beschränkten Gesamtwirkung“ \\ vor der aktienrechtlichen Dogmatik
}

\author{
Prof. Dr. Walter Bayer, Jena* \\ und \\ Dr. Philipp Scholz, Hamburg***
}

Inhaltsübersicht

ZGR 2016, 619-643

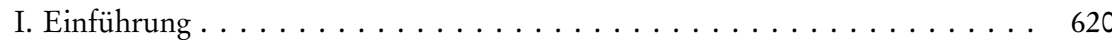

II. Auswirkungen des Arbeitnehmerhaftungsprivilegs auf die Organhaftung . . . . 621

1. Grundsätzlich gesamtschuldnerische Haftung von

Arbeitnehmern und Organmitgliedern . . . . . . . . . . . . . . . . 622

2. Interne Haftungsverteilung zwischen Arbeitnehmern

und Organmitgliedern . . . . . . . . . . . . . . . . . 623

3. Anspruchskürzung nach den Grundsätzen der „gestörten Gesamtschuld“? 625

4. Anspruchskürzung gemäß $\$ 254$ Abs. 2 Satz 2 BGB? . . . . . . . . . . 631

III. Auswirkungen von Verzicht und Vergleich gegenüber einem Organmitglied auf die mithaftenden Organmitglieder . . . . . . . . . . . . . .

2. Beschränkte Gesamtwirkung im Rahmen der aktienrechtlichen Organhaftung? . . . . . . . . . . . . . . . . . . . 634

3. Zulässigkeit einer Freistellungszusage für Regressansprüche der übrigen Organmitglieder? . . . . . . . . . . . . . . . . . . . . . 637

4. Konsequenz: Beschränkte Gesamtwirkung als rechtspolitisches Desiderat? 641

IV. Fazit . . . . . . . . . . . . . . . . . . . . . . . . . . . . . . . . . . . . . 642

Haftungserleichterungen gegenüber einem Gesamtschuldner werfen stets die Frage auf, ob sie auch zu Gunsten der Mithaftenden wirken, um eine Aushöhlung des Haftungsprivilegs im Wege des Gesamtschuldnerregresses zu verbindern. Dies gilt auch für die aktienrechtliche Organhaftung. Praktisch relevant sind dabei vor allem die gesamtschuldnerische Haftung von Organmitgliedern mit Arbeitnehmern bei Compliance-Verstößen sowie die Auswirkungen eines von der Hauptversammlung gebilligten Verzichts oder Vergleichs gegenüber einem Organmitglied. Ob es

* Inhaber des Lehrstuhls für Bürgerliches Recht, Handels- und Gesellschaftsrecht, Privatversicherungsrecht und Internationales Privatrecht an der Friedrich-Schiller-Universität Jena.

** Wissenschaftlicher Assistent bei Prof. Dr. Dr. h.c. mult. Reinhard Zimmermann am Max-Planck-Institut für ausländisches und internationales Privatrecht in Hamburg; vormals wissenschaftlicher Mitarbeiter am Lehrstuhl für Bürgerliches Recht, Handelsund Gesellschaftsrecht, Privatversicherungsrecht und Internationales Privatrecht an der Friedrich-Schiller-Universität Jena (Prof. Dr. Walter BAyer). 
in diesen Fällen trotz der aktienrechtlichen Verzichts- und Vergleichsbeschränkungen (\$ 93 Abs. 4 Satz 3 AktG) zu einer geräuschlosen Enthaftung der gesamtschuldnerisch mithaftenden Organmitglieder kommt, ist bislang kaum diskutiert. Der vorliegende Beitrag soll hierauf Antworten geben.

Joint and several liability generally raises the question whether privileging one debtor also affects the liability of the others as recourse by the non-privileged debtors would circumvent the liability privilege. This also applies to the liability of executive and supervisory board members in German stock corporations. The question arises most prominently in compliance cases, i.e. when board members are held liable for the wrongdoings of the company's employees. But it also arises when the corporation waives its claim against only one of several jointly liable board members. While general private law has long developed principles to deal with such cases, the premises of stock corporation law are quite different. Most importantly waiving a claim against a board member requires a resolution passed by the shareholders' meeting. This paper examines the quiet impact of the doctrine of joint and several liability on the board members' liability under the German Stock Corporation $\operatorname{Act}(A k t G)$.

\section{Einfübrung}

Die aktienrechtliche Organhaftung ist ebenso komplex wie streng. Sie sieht sich daher nicht nur dezidierter rechtspolitischer Kritik ausgesetzt. ${ }^{1}$ Auch wird - mit guten Gründen - dafür plädiert, dass sie bereits de lege lata $\mathrm{Be}$ schränkungen unterliegt, um zu verhindern, dass Vorstandsmitgliedern schon bei einfach fahrlässigen Pflichtverletzungen die wirtschaftliche Existenzvernichtung droht. ${ }^{2}$ Die in $\$ 93$ Abs. 2 Satz 1 AktG statuierte gesamtschuldnerische Haftung wird dabei vor allem als Teil des Problems wahrgenommen. ${ }^{3}$ Allerdings werfen Haftungsprivilegien zu Gunsten eines Gesamtschuldners bekanntlich Fragen nach den Auswirkungen auf die Ersatzpflicht der Mithaftenden auf. Wenngleich diese Fragen bis dato nicht im Fokus der aktienrechtlichen Diskussion standen, ${ }^{4}$ haben sie auch in diesem Kontext eine nicht unerhebliche Tragweite: Entfaltete etwa die zugunsten von Arbeitnehmern

1 Umfassend Bachmann, Gutachten E für den 70. Deutschen Juristentag, 2014, S. 28 ff; dazu etwa BAYER, NJW 2014, 2546; siehe auch die Beschlüsse der Abteilung Wirtschaftsrecht des 70. DJT vom 18.09.2014, ZIP 2014, 1902; zur Notwendigkeit einer Begrenzung der Vorstandshaftung BAYer/Scholz, NZG 2014, 926, 927 f; ausführlich Scholz, Die existenzvernichtende Haftung von Vorstandsmitgliedern, 2014, S. $227 \mathrm{ff}$.

2 Dezidiert Косн, AG 2014, 513 m.z.w.N.; zuletzt wieder Bayer/Scholz, GmbHR 2015, 449, 455 f; Норт/Rотн, Großkomm. z. AktG, 5. Aufl., 2015, $\$ 93$ Rdn. 398 ff; Schall, JZ 2015, 455; ausführlich Scholz, aaO (Fn. 1), S. 265 ff.

3 Etwa Bachmann, Gutachten E für den 70. Deutschen Juristentag, 2014, S. 42f, 122 (Empfehlung I.4.).

4 Siehe aber auch Voß, Gesamtschuldnerische Organhaftung, 2007. 
- außerhalb des Aufsichtsrats ${ }^{5}$ - eingreifende Haftungsbeschränkung bei betrieblich veranlasster Tätigkeit mittelbar auch Wirkung zugunsten mithaftender Vorstands- und Aufsichtsratsmitglieder, ist fraglich, was von der Compliance-Verantwortung letzterer überhaupt noch übrig bliebe. Gleichsinnig stellt sich die Frage, ob und wenn ja inwieweit ein nach $\mathbb{9} 93$ Abs. 4 Satz 3 AktG von der Hauptversammlung gebilligter Vergleich mit einem Vorstandsmitglied auch zur Enthaftung der anderen Mitglieder des Vorstands und des Aufsichtsrats führt.

Diese Zweifelsfragen gilt es im Folgenden aufzuklären. Dabei soll zunächst den Auswirkungen der beschränkten Arbeitnehmerhaftung - als anfängliche Privilegierung eines Gesamtschuldners - für die Fälle nachgegangen werden, in denen ein Schaden auf Pflichtverletzungen von Arbeitnehmern und Organmitgliedern zurückzuführen ist (sub II.). Sodann ist zu klären, inwiefern Verzicht und Vergleich gegenüber einem Organmitglied - als nachträgliche Privilegierung eines Gesamtschuldners - auch Wirkung zugunsten der mithaftenden Organmitglieder entfalten (sub III.).

\section{Auswirkungen des Arbeitnehmerhaftungsprivilegs auf die Organhaftung}

Lässt sich ein Schaden der Gesellschaft sowohl auf die Pflichtverletzung eines Arbeitnehmers als auch, wie bei ungenügender Compliance, auf Pflichtverletzungen der Mitglieder des Vorstands (und gegebenenfalls auch des Aufsichtsrats) zurückführen, stellt sich die Frage, ob die zugunsten des Arbeitnehmers eingreifenden Haftungsgrundsätze der betrieblich veranlassten Tätigkeit ${ }^{6}$ mittelbar eine Enthaftung der Organmitglieder bewirken. Hinter dieser Überlegung steht die - nach herrschender Lehre auf den innerbetrieblichen Schadensausgleich übertragbare ${ }^{7}$ - Rechtsprechung des BGH zur „gestörten Gesamtschuld“ im Falle der $\$ S$ 104, 105 SGB VII, wonach der

5 Henssler, Münchener Komm. z. BGB, 6. Aufl., 2012, \$619a Rdn. 19; LutTer/Krieger/ Verse, Rechte und Pflichten des Aufsichtsrats, 6. Aufl., 2014, $\mathbb{1} 13$ Rdn. 1024; SpIndLer,

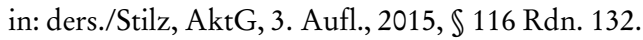

6 Grundlegend BAGE 78, 56; ausführlich zur historischen Entwicklung, zum persönlichen wie sachlichen Anwendungsbereich und zur Haftungsverteilung nach Verschuldensgrad nach Henssler, aaO (Fn. 5), $\$ 619$ a BGB Rdn. 5 ff; siehe auch Preis, Erfurter Komm. z. Arbeitsrecht, 16. Aufl., 2016, $\ 619$ a BGB Rdn. $12 \mathrm{ff}$.

7 Böttcher, in: Erman, BGB, 14. Aufl., 2014, $\$ 426$ Rdn. 35; Bydlinski, Münchener Komm. z. BGB, 7. Aufl., 2016, $\$ 426$ Rdn. 64; Looschelders, in: Staudinger, BGB, Neubearb. 2012, \426 Rdn. 169; STÜRnER, in: Jauernig, BGB, 16. Aufl., 2015, $\$ 426$ Rdn. 24. 
„Zweitschädiger in solchen Fällen in Höhe des Verantwortungsteils freigestellt [wird], der auf den Erstschädiger [scil.: Arbeitnehmer] im Innenverhältnis entfiele, wenn man seine Haftungsprivilegierung hinweg denkt" ${ }^{\text {“8 }}$.

Nach teilweise vertretener Auffassung sollen diese Grundsätze auch für den Fall eines Vorstands- oder Aufsichtsratsmitglieds als „Zweitschädiger“ Geltung beanspruchen können. ${ }^{9} \mathrm{Ob}$ dies tatsächlich überzeugt, bedarf jedoch nur dann näherer Erörterung, wenn die Organmitglieder mit den Arbeitnehmern im Grundsatz überhaupt gesamtschuldnerisch hafteten (hierzu sub 1.) und die Haftung im Innenverhältnis nicht ohnehin allein zu schultern hätten (hierzu $\operatorname{sub} 2$.).

\section{Grundsätzlich gesamtschuldnerische Haftung von Arbeitnehmern und Organmitgliedern}

Die Frage, ob Organmitglieder mit Angestellten der Gesellschaft als Gesamtschuldner haften, wenn ein Schaden der AG auf Pflichtverletzungen beider zurückzuführen ist, wird bis dato nur vereinzelt aufgeworfen, stets jedoch zu Recht bejaht. ${ }^{10}$ Denn insofern liegen nicht nur die geschriebenen Merkmale des $\$ 421 \mathrm{BGB}$ - eine Leistung, mehrere Schuldner - vor. Auch die ungeschriebenen Erfordernisse für die Annahme einer Gesamtschuld sind erfüllt: Zum

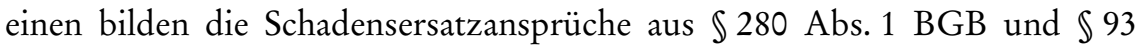
Abs. 2 AktG unschwer eine $Z$ weckgemeinschaft ${ }^{11}$, da beide kompensatorische wie präventive Ziele verfolgen. Zum anderen lässt sich im Verhältnis der Schadensersatzpflichten zueinander kein Stufenverhältnis ${ }^{12}$ ausmachen. ${ }^{13} \mathrm{Zwar}$ ist die Arbeitnehmerhaftung nach den Grundsätzen der betrieblich veranlassten Tätigkeit beschränkt und die Haftung der Organmitglieder gemäß $\$ 93$ Abs. 4 Satz 3 AktG nur mit Zustimmung der Hauptversammlung verzichtbar. Daraus lässt sich jedoch weder die Subsidiarität der Haftung des Arbeitnehmers ableiten noch der Schluss ziehen, dass die Organmitglieder primär für den Schaden aufkommen müssten. Denn beide Haftungsspezifika erschöpfen

8 BGH NJW 2008, 2116 Rdn. 11 m.w. N.

9 Mertens/Cahn, Kölner Komm. z. AktG, 3. Aufl., 2010, 993 Rdn. 51; zustimmend Норт/Roth, aаO (Fn. 2), $\$ 93$ AktG Rdn. 463 Fn. 1774; ebenso Voß, aaO (Fn. 4), S. $203 \mathrm{f}$.

10 Hopt/Roth, aaO (Fn. 2), $₫ 93$ AktG Rdn. 463; Mertens/Cahn, aaO (Fn. 9), $\$ 93$ AktG Rdn. 51; Voß, aaO (Fn. 4), S. 85.

$11 \mathrm{Zu}$ dieser in der früheren Rechtsprechung aufgestellten und mittlerweile wieder fallen gelassenen Voraussetzung siehe nur BYDLINSKI, aaO (Fn. 7), \421 BGB Rdn. 11 m.w. N.

$12 \mathrm{Zu}$ diesem Erfordernis ausführlich BGH NJW 2007, 1208 Rdn. $17 \mathrm{f} \mathrm{m.w.} \mathrm{N.;} \mathrm{BYdLINski,}$

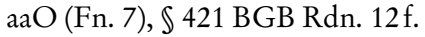

13 Ebenso, wenngleich ohne Begründung, $\mathrm{Voß}$, aaO (Fn. 4), S. 85. 
sich in einer Regelung des Verhältnisses zur Gesellschaft: Der arbeitsrechtlichen Haftungsprivilegierung liegt der Gedanke zu Grunde, dass eine vollständige Ersatzpflicht des Arbeitnehmers angesichts des dem Arbeitgeber - i.e. der Gesellschaft - zuzurechnenden Betriebsrisikos sowie dessen Befugnis zur Organisation des Betriebs und zur Gestaltung der Arbeitsbedingungen nicht angemessen ist. ${ }^{14}$ Auch $\ 93$ Abs. 4 Satz 3 AktG beschränkt sich nach seiner ratio legis auf eine Regelung im alleinigen Interesse der aktienrechtlichen Corporate Governance. ${ }^{15}$

\section{Interne Haftungsverteilung zwischen Arbeitnebmern und Organmitgliedern}

Haften also Arbeitnehmer und Organmitglieder im Grundsatz, also vorbehaltlich des arbeitsrechtlichen Haftungsprivilegs, als Gesamtschuldner, stellt sich die Frage nach der internen Haftungsverteilung i.S.d. $\$ 426$ Abs. 1 Satz 1 BGB. Denn müssten die Organmitglieder im Innenverhältnis (zum Arbeitnehmer) den ganzen Schaden tragen, käme eine Kürzung der Haftung im Außenverhältnis (zur Gesellschaft) nach den Grundsätzen der gestörten Gesamtschuld ohnehin nicht in Betracht.

Nach den zur Gleichrangigkeit der Haftung von Arbeitnehmern und Organmitgliedern angestellten Überlegungen ${ }^{16}$ lässt sich eine alleinige Haftungsverantwortlichkeit der Organmitglieder jedenfalls weder aus der Beschränktheit der Arbeitnehmerhaftung ableiten noch unter Rekurs auf $\$ 93$ Abs. 4 Satz 3 AktG begründen. $\mathrm{Da}$ es somit an einem speziellen Verteilungsschlüssel fehlt, muss auf allgemeine Grundsätze zurückgegriffen werden. Dementsprechend ist nach dem Rechtsgedanken des $\$ 254$ BGB vornehmlich auf die Verursachungsbeiträge abzustellen. ${ }^{17}$ Sieht man einmal von den Fällen einer enthaftenden und damit eine Gesamtschuld ohnehin ausschließenden Weisung des Vorstands an den Arbeitnehmer ab, kommt eine gesamtschuldnerische Haftung von Arbeitnehmern und Organmitgliedern vor allem bei Compliance-Verstößen in Betracht. Diese Fälle sind durch ein rechtswidriges Verhalten von Arbeitnehmern geprägt, das durch zumutbare organisatorische Vorkehrungen

14 BAGE 78, 56 (64).

15 Zu den Normzwecken des $₫ 93$ Abs. 4 Satz 3 AktG siehe nur BAYER/Scholz, ZIP 2015, $149,150 f$.

16 Oben II.1.

17 In diesem Sinne die ständige Rechtsprechung des BGH, aus jüngerer Zeit etwa BGH NJW 2011, 292 Rdn. 9; BGH NJW 2006, 896 Rdn. 12; siehe ferner BYDLINSKI, aaO (Fn. 7), \426 BGB Rdn. 21; GeHrLein, in: BeckOK, BGB, 39. Ed., 2016, $\$ 426$ Rdn. 9. 
des Vorstands, insbesondere die Veranlassung entsprechender Überwachung, hätte verhindert werden können. ${ }^{18}$

In der bürgerlich-rechtlichen Auseinandersetzung mit $\$ 426$ BGB werden derlei Konstellationen unter dem nicht ganz präzisen Schlagwort der „Haftung für fremdes Verschulden“ behandelt, wobei - allen voran für die gesamtschuldnerische Haftung von Geschäftsherr und Verrichtungsgehilfe in den Fällen des $₫ 831$ BGB - aus der Regelung des $₫ 840$ Abs. 2 BGB das Prinzip gewonnen wird, dass derjenige, der nach außen für fremdes Verschulden einzustehen hat, im Innenverhältnis frei sein soll. ${ }^{19}$ Ließe man diese Grundregel auch im hiesigen Zusammenhang zur Anwendung gelangen, hätte der Arbeitnehmer den Schaden im Innenverhältnis allein zu tragen, wenn nicht den Organmitgliedern ausnahmsweise ein deutlich größeres Verschulden - sprich: grobe Fahrlässigkeit oder Vorsatz - zur Last fiele. ${ }^{20}$ In der Tat lässt sich eine Parallele zur gesamtschuldnerischen Haftung von Geschäftsherr und Verrichtungsgehilfe im Falle des $₫ 831$ Abs. 1 BGB nicht von der Hand weisen. ${ }^{21}$ Allerdings dürfte sich ein vorbehaltloser Rekurs auf diesen Grundsatz des Gesamtschuldnerausgleichs dem Vorwurf ausgesetzt sehen, die aktienrechtlichen Besonderheiten aus dem Blick zu lassen, insbesondere dass mit der Compliance-Verantwortung eine spezifisch organschaftliche Pflicht in Rede steht.

Hier hilft ein vergleichender Blick auf die interne Haftungsverteilung zwischen Mitgliedern des Vorstands und des Aufsichtsrats in den Fällen, in denen sich die Vorstandsmitglieder wegen einer unmittelbaren Sorgfaltspflichtverletzung haftbar gemacht haben und die Haftung der Aufsichtsratsmitglieder auf einem Überwachungsverschulden gründet. Diese Konstellation ist nicht nur ob der in Rede stehenden Pflichtverletzungen vergleichbar. Vor allem steht auch hier die Verletzung einer organschaftlichen Überwachungspflicht in Rede. Darüber hinaus handelt es sich bei dieser sogar um die primäre Pflicht des Aufsichtsrats als Überwachungsorgan. Der Verstoß gegen die Überwachungs-

$18 \mathrm{Vgl}$. etwa Hopt/Roth, aaO (Fn. 2), $\$ 93$ AktG Rdn. 182: Vorstandsmitglied muss „dafür Sorge tragen, dass das Unternehmen so organisiert und beaufsichtigt wird, dass keine Gesetzesverstöße erfolgen“.

19 Bydlinski, aaO (Fn. 7), \426 BGB Rdn. 21; Gehrlein, aaO (Fn. 17), 4426 BGB Rdn. 9; STÜRNER, aaO (Fn. 7), $\$ 426$ BGB Rdn. 9.

20 Für gleiche Haftungsanteile dagegen Voß, aaO (Fn. 4), S. 203, der die von ihm ermittelten Haftungsquoten indes abstrakt und nicht im Hinblick auf Compliance-Verstöße erörtert.

21 Freilich ist Geschäftsherr des Arbeitnehmers i.S.d. $\int 831$ Abs. 1 BGB nicht der Vorstand, sondern die Gesellschaft. Wertungsmäßig geht es indes um die Frage der Verantwortlichkeit im Innenverhältnis und insofern sind die Situationen durchaus vergleichbar, weil bei Compliance-Verstößen der Arbeitnehmer wie der Geschäftsführer den Schaden unmittelbar verursacht und dem Vorstand wie dem Geschäftsherrn nur ein Überwachungsverschulden (i. w. S.) anzulasten ist. 
aufgabe dürfte daher im Vergleich sogar tendenziell noch schwerer wiegen. Indes zieht die ganz herrschende Auffassung auch hier die Wertung des $\$ 840$ Abs. 2 BGB heran und lässt die Vorstandsmitglieder den Schaden daher im Innenverhältnis grundsätzlich alleine tragen. ${ }^{22}$

Dementsprechend wird sich auch im Verhältnis von Arbeitnehmer- und Vorstandshaftung schwerlich einwenden lassen, dass die Verletzung der organschaftlichen Pflichten eine Abweichung von den Wertungen des $\$ 840$ Abs. 2 BGB erforderlich mache und trotz der "Haftung für fremdes Verschulden“ eine anteilige Schadenstragung des Vorstands im Innenverhältnis gebiete. In den klassischen Compliance-Fällen trifft den Arbeitnehmer daher im Innenverhältnis grundsätzlich die volle Haftungsverantwortlichkeit.

\section{Anspruchskürzung nach den Grundsätzen der "gestörten Gesamtschuld“?}

Der Frage, ob die Organhaftung nach den Grundsätzen der „gestörten Gesamtschuld" zu kürzen ist, kommt mithin eine ganz erhebliche Brisanz zu. Da der Arbeitnehmer bei Compliance-Verstößen den Schaden im Innenverhältnis regelmäßig allein zu tragen hätte, würde das arbeitsrechtliche Haftungsprivileg vollständig auf die Organhaftung durchschlagen. Die vollständige Enthaftung des Arbeitnehmers entließe mithin auch die Organmitglieder aus der Haftung. Im Übrigen würde sich deren Ersatzpflicht auf die nach den Verhältnissen des Arbeitnehmers bemessene Haftungssumme beschränken, was einer vollständigen Enthaftung in der Regel faktisch gleichkäme. Von der Haftung für Compliance-Verstöße bliebe damit wenig bis gar nichts übrig. Diese Konsequenz wird im Schrifttum indes bislang nicht gezogen, soweit einer Begrenzung der Organhaftung nach den Grundsätzen der „gestörten Gesamtschuld“ das Wort

22 Fleischer, in: Spindler/Stilz, AktG, 3. Aufl., 2015, \93 Rdn. 263; Grigoleit/Tomasic, in: Grigoleit, AktG, 1. Aufl., 2013, $₫ 93$ Rdn. 66; Hölters, in: ders., AktG, 2. Aufl., 2014, \93 Rdn. 247; für grundsätzlich alleinige Verantwortlichkeit auch ohne Rekurs auf $\ 840$ Abs. 2 BGB Hорт/Roth, aaO (Fn. 2), $\ 93$ AktG Rdn. 465; ebenso für die gesamtschuldnerische Haftung mehrerer Vorstandsmitglieder, wenn diesen teils nur ein Überwachungsverschulden gegenüber den unmittelbar Verantwortlichen zur Last fällt, Bürgers/Israel, in: Bürgers/Körber, AktG, 3. Aufl., 2014, $₫ 93$ Rdn. 31; Fleischer, aaO, $\mathbb{9} 93$ AktG Rdn. 263; Норт/Rотн, aaO (Fn. 2), $\mathbb{9} 93$ AktG Rdn. 465; so auch die h.M. für die GmbH-Geschäftsführerhaftung: Fleischer, Münchener Komm. z. GmbHG, 2. Aufl., 2016, $₫ 43$ Rdn. 319 m.w.N.; Hans/Ziemons, in: Michalski, GmbHG, 2. Aufl., 2010, $\mathbb{} 43$ Rdn. 228; Oetker, in: Henssler/Strohn, GesR, 3. Aufl.,

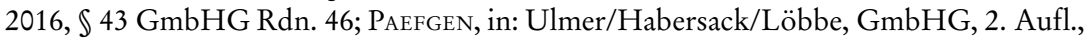
2014, Rdn. 200; Scholz/Schneider/Crezelius, Komm. z. GmbHG, 11. Aufl., 2013, Rdn. 252; Zöllner/Noack, in: Baumbach/Hueck, GmbHG, 20. Aufl., 2013, \43 Rdn. 29; a.A. aber Habersack, Münchener Komm. z. AktG, 4. Aufl., 2014, $\mathbb{1} 116$ Rdn. 73. 
geredet wird, wenn Organmitglieder neben Mitarbeitern für den Schaden verantwortlich sind. ${ }^{23}$

Tatsächlich gibt nicht nur diese weitgehende Enthaftung der Vorstands- und Aufsichtsratsmitglieder Anlass zu Bedenken. Vielmehr stellt sich grundsätzlich die Frage, ob es für die strenge aktienrechtliche Organhaftung die angemessene und dogmatisch überzeugende Lösung ist, die „gestörte Gesamtschuld“ zu Lasten der Gesellschaft aufzulösen.

\section{a) Alternativen zur Auflösung der, gestörten Gesamtschuld“ zu Lasten der Gesellschaft}

Die Auflösung zu Lasten der Gesellschaft ist jedenfalls nicht die einzige und schon gar nicht die gesetzlich zwingende Alternative zur Auflösung der Problematik.

Nimmt man den Wortlaut des $₫ 426$ Abs. 1 BGB ernst, so besteht nämlich schon keine Gesamtschuld, wenn und soweit der Arbeitnehmer von der Haftung freigestellt ist. Nach diesem Ansatz, den der BGH mittlerweile etwa für die Haftungsprivilegierung des $\$ 1664$ Abs. 1 BGB verfolgt, ${ }^{24}$ wird die „gestörte Gesamtschuld“ $z$ L Lasten des Zweitschädigers aufgelöst: Die Organmitglieder hafteten danach unbeschränkt, ohne dass die Möglichkeit eines Rückgriffs bestünde.

Alternativ hierzu kommt zumindest theoretisch eine Auflösung zu Lasten des Arbeitnehmers in Betracht, wenn man von der Prämisse ausgeht, dass dessen Haftungserleichterung nicht zu Lasten Dritter gehen soll. Hiernach wäre allein für den Zweck des Gesamtschuldnerausgleichs eine gleichsam „hinkende“ Gesamtschuld zu fingieren, um dem Organmitglied einen Rückgriff gegenüber dem Arbeitnehmer zu ermöglichen. ${ }^{25}$ Die Rechtsprechung favorisiert diese Lösungsvariante für zwischen Erstschädiger und Geschädigtem vereinbarte Haftungsprivilegien, weil das vertragliche Haftungsprivileg sonst einem unzulässigen Vertrag zu Lasten Dritter, namentlich des Zweitschädigers, gleichkäme. ${ }^{26}$

Derlei Bedenken gegen eine Auflösung zu Lasten der Organmitglieder als Zweitschädiger bestehen - so viel sei vorweggenommen - im Hinblick auf die

23 Vgl. Mertens/Cahn, aaO (Fn. 9), $\$ 93$ AktG Rdn. 51; Voß, aaO (Fn. 4), S. 203 f.

24 BGHZ 103, 338.

25 JANDA, VersR 2012, 1078, 1079; auf dieser Grundlage stellte sich allerdings die Frage, ob dem Arbeitnehmer nach der ratio der Haftungsprivilegierung nicht wiederum ein Rückgriffsanspruch gegen die Gesellschaft einzuräumen wäre, was wirtschaftlich wiederum auf eine Auflösung der „gestörten Gesamtschuld“ zu Lasten der Gesellschaft hinausliefe (vgl. Gehrlein, aaO (Fn. 17), \426 BGB Rdn. 12).

26 BGH NJW 1989, 2386, 2387; BGHZ 58, 216, 220; BGHZ 12, 213. 
Haftungserleichterung bei betrieblich veranlasster Tätigkeit jedenfalls nicht. Denn diese beruht nicht auf Vereinbarung, sondern wurde vom BAG unter Heranziehung von $\$ 254$ BGB in richterlicher Rechtsfortbildung entwickelt. ${ }^{27}$ Sie versteht sich also als ein gleichsam gesetzliches Haftungsprivileg. Ein solches kann freilich auch zu Lasten des Zweitschädigers wirken. Ob dieser durch eine Anspruchskürzung im Außenverhältnis mittelbar privilegiert oder durch Verhinderung des Gesamtschuldnerregresses benachteiligt werden soll, ist somit vor allem eine Wertungsfrage. Dabei liegt es sogar nahe, die „gestörte Gesamtschuld“ im Zweifel zu Lasten des Zweitschädigers aufzulösen. Denn für eine gesetzesübersteigende Anspruchskürzung bleibt nach allgemeinen Grundsätzen eigentlich kein Raum, wenn die ratio der Haftungsprivilegierung für die Frage der Belastung bzw. Privilegierung des Zweitschädigers unergiebig ist. ${ }^{28}$

\section{b) Tragende Gründe für die Auflösung zu Lasten des Arbeitgebers außerhalb der Organhaftung}

Nach dem BGH beruht die Auflösung der „gestörten Gesamtschuld“ zu Lasten des Arbeitgebers im Falle der Haftungsfreistellung der $\$ \$$ 104, 105 SGB VII auf der Überlegung,

„dass einerseits die haftungsrechtliche Privilegierung nicht durch eine Heranziebung im Gesamtschuldnerausgleich unterlanfen werden soll, es aber andererseits bei Mitberücksichtigung des Grundes der Haftungsprivilegierung, nämlich der anderweitigen Absicherung des Geschädigten durch eine gesetzliche Unfallversicherung nicht gerechtfertigt wäre, den Zweitschädiger den Schaden alleine tragen zu lassen. " ${ }^{29}$

Obschon diese Rechtsprechung nach der herrschenden Auffassung im Schrifttum auch für das Haftungsprivileg der betrieblich veranlassten Tätigkeit gelten soll, ${ }^{30}$ lassen sich die Gründe für eine Auflösung zu Lasten des Arbeitgebers nicht eins zu eins übertragen. Zwar verfängt der Einwand, die haftungsrecht-

27 Vgl. BAGE 78, 56 (63).

28 Anders aber Costede, JR 2005, 45, 49f mit dem Argument, nicht die Teilschuld, sondern die Gesamtschuld stelle die begründungsbedürftige Ausnahme dar. Doch beruht dies auf der unzureichenden Grundannahme (Costede, JR 2005, 45, 48), dass die Teilschuld die konsequente Lösung sei, weil der Schadensersatz dem Ausgleich einer Schädigung diene und sich dessen Umfang daher nach dem Schadensumfang und nicht nach der Anzahl der Schädiger. Letzteres ist zwar für sich genommen richtig. Indes darf der Schadensumfang nicht mit den Verantwortlichkeitsbeiträgen gleichgesetzt werden. $\mathrm{Zu}$ einer (gestörten) Gesamtschuld kommt es ohnehin nur, soweit der Schaden nach allgemeinen schadensrechtlichen Grundsätzen dem Verhalten beider Schädiger zurechenbar ist.

29 BGH NJW 2008, 2116 Rdn. 11.

30 Böttcher, aaO (Fn. 7), \426 BGB Rdn. 35; Bydlinski, aaO (Fn. 7), \426 BGB Rdn. 64; Looschelders, aaO (Fn. 7), $\$ 426$ BGB Rdn. 169; StÜrner, aaO (Fn. 7), $\$ 426$ BGB Rdn. 24. 
liche Privilegierung des Arbeitnehmers dürfe im Wege des Gesamtschuldnerausgleichs nicht umgangen werden. Den Haftungsgrundsätzen der betrieblich veranlassten Tätigkeit fehlt es jedoch an der "anderweitigen Absicherung des Geschädigten" durch eine Versicherung. ${ }^{31}$ Damit mangelt es insoweit an dem für die Belastung des Geschädigten maßgeblichen Begründungsaspekt. Eine

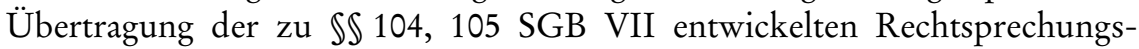
grundsätze ist daher bereits im Ansatz nicht frei von Zweifeln.

\section{c) Auflösung zu Lasten der Organmitglieder}

Auch bei unvoreingenommener Betrachtung der „gestörten Gesamtschuld“ zwischen Arbeitnehmern und Organmitgliedern muss im Ausgangspunkt feststehen, dass die ratio des arbeitsrechtlichen Haftungsprivilegs eine Auflösung zu Lasten der Arbeitnehmer verbietet. Dementsprechend verbleiben als Alternativen lediglich die Auflösung zu Lasten der Gesellschaft oder der Organmitglieder.

Dabei spricht für eine Belastung der Gesellschaft zunächst recht offensichtlich, dass den Organmitgliedern im praktischen Regelfall des Compliance-Verstoßes gerade nicht entgegengehalten werden kann, sie hätten den Schaden ohne die Mithaftung des Arbeitnehmers auch alleine tragen müssen. Denn die unzureichende Überwachung hätte es für sich genommen keinesfalls vermocht, den Schaden zu begründen. Erst durch das unmittelbar schadensbegründende Handeln des Arbeitnehmers wird aus dem Compliance-Verstoß ein Haftungsfall. Eine Enthaftung der Organmitglieder wäre für diese also gerade kein windfall profit aus der zufälligen Mitverantwortlichkeit eines weiteren Schädigers.

Umgekehrt zeigt gerade diese Argumentation, dass von der Compliance-Verantwortung der Organe wenig bis gar nichts verbliebe, wenn Vorstand und Aufsichtsrat insoweit praktisch keine Haftung zu befürchten hätten. Nun mag man versucht sein, gegen derlei - unter Präventionsgesichtspunkten berechtigte - Anreizüberlegungen einzuwenden, dass der aktienrechtlichen Organhaftung keine Straffunktion zukommt. Dieser Einwand ist hier jedoch nicht angebracht. Denn bei einer Vollhaftung wären die Organmitglieder auch nur zum Ersatz des tatsächlich entstandenen Schadens verpflichtet. Spiegelbildlich würde die Gesellschaft durch eine Versagung der Anspruchskürzung nicht besser, sondern lediglich nicht schlechter gestellt.

Es ist zwar nicht von der Hand zu weisen, dass den Organmitgliedern in Ermangelung einer Gesamtschuld der Rückgriff bei dem unmittelbar verant- 
wortlichen Arbeitnehmer versagt wäre. Indes geht die Erwartung, die Organe könnten sich auf diesem Wege im Ergebnis schadlos halten, an der Realität vorbei. ${ }^{32}$ Der Gesamtschuldnerregress würde regelmäßig nur zur Privatinsolvenz der Arbeitnehmer führen. Mit Ausnahme der Existenzvernichtung des Arbeitnehmers entspricht eine Belastung der Organmitglieder somit wirtschaftlich der Auflösung der Gesamtschuld, wenn diese nicht durch das arbeitsrechtliche Haftungsprivileg "gestört" wäre.

Aus dieser Perspektive wäre eine Anspruchskürzung nach den Grundsätzen der „gestörten Gesamtschuld“ tatsächlich regelmäßig ein Zufallsgeschenk für die Organmitglieder. Das gilt, da die Versicherung der Haftung folgt, freilich ebenso für die D\&O-Versicherer. Der Gesellschaft kämen mithin nicht nur die Organmitglieder als Schuldner abhanden, sondern auch ein etwaig bestehender Versicherungsschutz, wenngleich sich diesem Problem durch entsprechende Vertragsgestaltung Herr werden ließe.

Darüber hinaus begegnet die Kürzung des Anspruchs gegen haftpflichtige Organmitglieder auch mit Blick auf die Strenge der aktienrechtlichen Organhaftung Bedenken: Es passt schwerlich zusammen, dass die Haftung einerseits unverhandelbar unbeschränkt ist und nur unter den Voraussetzungen des $\ 93$ Abs. 4 Satz 3 AktG erlassen werden kann, andererseits ausgerechnet auf Grund des Arbeitnehmerhaftungsprivilegs gemildert sein soll. Freilich mag man hiergegen einwenden, dass die Gesamtschuldproblematik rechtskonstruktiv den Verzichts- und Vergleichsbeschränkungen vorgelagert ist, weil Ersatzansprüche eben nur in dem Umfang bestehen, wie sie sich aus den gesetzlichen Regelungen ergeben. Allerdings müsste dann auch der Einwand Gehör finden, dass rechtskonstruktiv bei Eingreifen des arbeitsrechtlichen Haftungsprivilegs schon gar keine Gesamtschuld besteht. Die Auflösung der ,gestörten Gesamtschuld" ist vielmehr ein Wertungsproblem und auch als solches zu behandeln. Daher ist die Strenge der aktienrechtlichen Organhaftung sehr wohl ein ernst zu nehmendes Datum, wobei nicht nur $\$ 93$ Abs. 4 Satz 3 AktG, sondern auch \93 Abs. 2 Satz 3 AktG die Notwendigkeit eines persönlichen Haftungsrisikos unter Präventionsgesichtspunkten unterstreicht.

In diesem Sinne kann schließlich nicht außer Acht gelassen werden, dass die Grundsätze der betrieblich veranlassten Tätigkeit auf Organmitglieder - auch auf die Arbeitnehmervertreter im Aufsichtsrat ${ }^{33}$ - gerade keine Anwendung

32 Dieses Argument ist im vorliegenden Kontext folglich ebenso deplatziert wie im Falle des Bußgeldregresses gegenüber Geschäftsleitern, wenn dort eingewandt wird, die Regressmöglichkeit würde die Entscheidung des Gesetzgebers konterkarieren, das Unternehmen zur Verantwortung zu nehmen (vgl. bereits BAYER/SchOlz, GmbHR 2015, 449, 452).

33 Zur Identität der Verantwortlichkeit aller Aufsichtsratsmitglieder siehe nur HABERSACK,

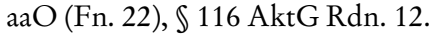


finden ${ }^{34}$ und sich den ihnen zugrunde liegenden Wertungen auch nicht entnehmen lässt, dass sie eine mittelbare Begünstigung Dritter verlangen. Insbesondere kommt den Aspekten des Betriebsrisikos, der Weisungsgebundenheit des Arbeitnehmers sowie der Befugnis des Arbeitgebers zur Organisation des Betriebs nur zwischen Arbeitnehmer und Arbeitgeber Relevanz zu. Im Verhältnis zwischen Organmitgliedern und Gesellschaft, wo nicht einmal konkrete Pflichtverletzungen von Mitgliedern der Unternehmensleitung den Mitverschuldenseinwand begründen können, ${ }^{35}$ spielen sie erst recht keine Rolle. Vielmehr üben die Vorstandsmitglieder in der Aktiengesellschaft gerade umgekehrt die Arbeitgeberfunktion aus. ${ }^{36}$

In summa bleibt damit festzuhalten, dass (i) die Grundsätze der betrieblich veranlassten Tätigkeit eine Auflösung der „gestörten Gesamtschuld“ zu Lasten der Gesellschaft nicht erfordern, (ii) dieser Weg auch schwerlich mit der Strenge der aktienrechtlichen Organhaftung vereinbar ist, (iii) eine Auflösung zu Lasten der Organmitglieder unter Präventionsgesichtspunkten angezeigt ist und (iv) eine Versagung der Anspruchskürzung die Organmitglieder auch nicht unbillig belasten würde. Die „gestörte Gesamtschuld“ von Arbeitnehmern und Organmitgliedern ist daher nicht zu Lasten der Gesellschaft, sondern zu Lasten der Organmitglieder aufzulösen. Mithin ist der Anspruch der Gesellschaft gegen letztere nicht um den Verantwortungsteil des Arbeitnehmers zu kürzen.

\section{d) Keine abweichende Beurteilung auf Grundlage einer de lege lata beschränkten Vorstandshaftung}

An dieser Beurteilung änderte sich auch dann nichts, wenn man mit der mittlerweile wohl herrschenden Auffassung im Schrifttum ${ }^{37}$ davon ausgeht, dass die aktienrechtliche Organhaftung ihrerseits bereits de lege lata Beschränkungen unterliegt, um zu verhindern, dass den Organmitgliedern schon bei einem fahrlässigen Pflichtverstoß die wirtschaftliche Existenzvernichtung droht. Denn legt man diese Haftungsreduktion zu Grunde, ist es sogar noch weniger

34 BGH WM 1975, 467, 469; Норт/Roth, aаO (Fn. 2), \93 AktG Rdn. 395 ff m.z.w.N.; ausführlich Scholz, aaO (Fn. 1), S. 267 f.

35 Siehe nur Fleischer, aaO (Fn. 22), $\$ 93 \mathrm{AktG}$ Rdn. 210; Норt/Roth, aaO (Fn. 2), $\$ 93$ AktG Rdn. 404; zur GmbH-Geschäftsführerhaftung eingehend BAYER/SCHOLZ, GmbHR 2016, 841, $842 \mathrm{f}$.

36 Koch, in: Hüffer/Koch, AktG, 12. Aufl., 2016, $\$ 84$ Rdn. 14.

37 Siehe nur Bayer/Scholz, NZG 2014, 926, 928; КосH, AG 2014, 513 (jeweils m.w. N.);

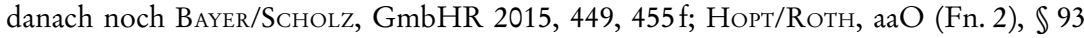
AktG Rdn. 398 ff; Schall, JZ 2015, 455; ausführlich Scholz, aaO (Fn. 1), S. 265 ff; a. A. Fleischer, ZIP 2014, 1305, 1307 f; Schöne/Petersen, AG 2012, 700, $700 \mathrm{ff}$. 
bedenklich, die „gestörte Gesamtschuld“ zu Lasten der Organmitglieder aufzulösen. Umgekehrt würden die spezifischen Grenzen der aktienrechtlichen Haftungsbegrenzung bei einer Anspruchskürzung nach den Grundsätzen der „gestörten Gesamtschuld“ ausgehöhlt. Eine Auflösung zu Lasten der Organmitglieder wäre folglich a fortiori geboten. ${ }^{38}$

\section{Anspruchskürzung gemäß 254 Abs. 2 Satz $2 B G B$ ?}

Soweit die Begründungslücke bei der Übertragung der Rechtsprechung zu \\104, 105 SGB VII auf die Haftungsgrundsätze der betrieblich veranlassten Tätigkeit erkannt wird, ${ }^{39}$ versucht das bürgerlich-rechtliche Schrifttum diese unter Hinweis darauf zu schließen, dass sich der Arbeitgeber gegenüber dem Zweitschädiger gemäß $\ 254$ Abs. 2 Satz 2 BGB i.V.m. $\ 278$ BGB das Verschulden seines Arbeitnehmers bei der Schadensentstehung zurechnen lassen müsse. ${ }^{40}$ Allerdings ist das Haftungsprivileg des Arbeitnehmers für diesen Weg der Anspruchskürzung ohne Belang. Mit diesem Einwand wird mithin die Diskussion um die angemessene Auflösung der "gestörten Gesamtschuld“ verlassen und die grundsätzliche, indes wenig diskutierte Frage nach dem Verhältnis zwischen $\ 254$ Abs. 2 Satz 2 BGB und den Regeln der (gestörten) Gesamtschuld aufgeworfen. ${ }^{41}$ Für den Fall der gemeinsamen Schadensverantwortlichkeit von Arbeitnehmern und Organmitgliedern bedarf es hierauf jedoch keiner Antwort. Richtigerweise wäre es den Organmitgliedern nämlich auch dann verwehrt, der Gesellschaft das Mitverschulden der Arbeitnehmer entgegenzuhalten, wenn man $\$ 254$ Abs. 2 Satz 2 BGB in den Konstellationen der „gestörten Gesamtschuld“ für anwendbar hielte. Das folgt aus einem argumentum a maiore ad minus aus der in $\$ 93$ Abs. 2 Satz 1 AktG angeordneten Haftung der Organmitglieder als Gesamtschuldner. Diese derogiert in ihrem unmittelbaren Anwendungsbereich die Möglichkeit, das Mitverschulden eines anderen Gesellschaftsorgans oder Organmitglieds gemäß $\$ \$ 254,31$ BGB gegenüber der Gesellschaft einzuwenden. ${ }^{42}$ Sie ist damit letztlich Kon-

38 So auch für die gestörte Gesamtschuld unter Organmitgliedern bei Annahme einer aktienrechtlichen Haftungsprivilegierung Scholz, aaO (Fn. 1), S. $339 \mathrm{ff}$; a. A. Brommer, AG 2013, 121, 130; CASPER, ZHR 176 (2012), 617, $643 \mathrm{f}$.

39 Dazu oben II.3.b).

40 JANDA, VersR 2012, 1078, 1085.

41 Für einen Vorrang der Regeln der „gestörten Gesamtschuld“ Hager, NJW 1989, 1640, 1645; für eine restriktive Interpretation des $\$ 254$ Abs. 2 Satz 2 BGB gegenüber den Regeln der Gesamtschuld auch RüßMAnN, in: jurisPK, BGB, 7. Aufl., 2014, $\mathbb{2} 254$ Rdn. 27.

42 Siehe nur Fleischer, aaO (Fn. 22), $₫ 93$ AktG Rdn. 210; zu $₫ 43$ Abs. 2 GmbHG auch BGH ZIP 1983, 824, 825; zu den Grenzen der Verdrängung des Mitverschuldenseinwandes BAYER/SCHOLZ, GmbHR 2016, 841, $842 \mathrm{f}$. 
sequenz der Gesamtverantwortung der Organmitglieder. ${ }^{43}$ Setzt sich aber nach dem Grundsatz der Gesamtverantwortung im Verhältnis zur Gesellschaft die individuelle Haftungsverantwortung der Organmitglieder sogar gegenüber der Verantwortlichkeit gleichberechtigter Organmitglieder durch, muss es ihnen erst recht verwehrt sein, sich auf das Mitverschulden von in die Organisation eingegliederten und den Weisungen der Geschäftsleitung unterworfenen Angestellten zu berufen. Das wird am Beispiel des Überwachungsverschuldens der Aufsichtsratsmitglieder besonders deutlich: Wenn es für deren Haftung insoweit sogar ohne Belang ist, dass der Vorstand, welcher die Gesellschaft gemäß $\$ 76$ Abs. 1 AktG unter eigener Verantwortung leitet, für die Pflichtverletzung unmittelbar verantwortlich ist, kann es schwerlich zur Anspruchskürzung führen, wenn die unmittelbare Verantwortung bei einem Angestellten der Gesellschaft liegt und die Vorstandsmitglieder ebenfalls nur ein Überwachungsverschulden trifft.

\section{Auswirkungen von Verzicht und Vergleich gegenüber einem Organmitglied auf die mithaftenden Organmitglieder}

Die gesamtschuldnerische Haftung von Organmitgliedern wirft indes nicht nur Fragen auf, wenn sie durch anfängliche Haftungsprivilegien "gestört" ist. Zwar mag die Rechtslage auf den ersten Blick recht klar erscheinen, wenn der Gesellschaft ausschließlich Organmitglieder als Gesamtschuldner haften. Denn eine Begrenzung der Ersatzansprüche ist dann nur unter den Voraussetzungen des $₫ 93$ Abs. 4 Satz 3 AktG möglich. Kommen Vergleich oder Verzicht jedoch nur gegenüber einzelnen Organmitgliedern wirksam zustande, genügt ein flüchtiger Blick ins Aktiengesetz nicht mehr, um die Haftungsverhältnisse nachzuvollziehen. Hier stellt sich im Grunde dieselbe Frage wie bei der gesamtschuldnerischen Haftung von Organmitgliedern und Arbeitnehmern, nämlich, ob eine Entlastung eines Gesamtschuldners mittelbar die Enthaftung der anderen bewirkt, weil Verzicht und Vergleich andernfalls im Wege des Gesamtschuldnerregresses drohten, konterkariert zu werden.

\section{Auswirkungen von Verzicht und Vergleich auf die Haftung der übrigen Gesamtschuldner nach bürgerlich-rechtlichen Grundsätzen}

Der Gesamtschulddogmatik ist das Problem freilich wohlbekannt, wobei es in der dort geläufigen Diktion um die Frage geht, ob einem Verzicht bzw. Vergleich „Einzelwirkung“ oder „Gesamtwirkung“ zukommt. Die einschlägige Regelung des $\ 423$ BGB enthält sich zwar einer Lösung des Problems. Sie 
macht jedoch deutlich, dass es sich um eine Frage der Auslegung der Abrede zwischen Gläubiger und privilegiertem Gesamtschuldner handelt. Dabei steht im Ausgangspunkt zweierlei fest: Will der Gläubiger den einzelnen Gesamtschuldner endgültig freistellen, ist dies zum einen nur zu seinen eigenen Lasten und nicht zu Lasten der am Erlassvertrag unbeteiligten Gesamtschuldner möglich. ${ }^{44}$ Insofern verhält es sich wie bei vertraglichen Haftungserleichterungen im Rahmen der gestörten Gesamtschuld. ${ }^{45}$ Zum anderen werden Verzicht und Vergleich in der Gesamtschulddogmatik - wie auch im Rahmen des $\$ 93$ Abs. 4 Satz 3 AktG - im Grundsatz gleich behandelt. ${ }^{46}$

\section{a) Gesamtwirkung, Einzelwirkung und beschränkte Gesamtwirkung}

Dementsprechend können Verzicht und Vergleich gegenüber einem Gesamtschuldner folgende Wirkungen für die Haftung der übrigen Gesamtschuldner zeitigen:

So kann als Sonderfall eines zulässigen Erlassvertrages zugunsten Dritter ${ }^{47}$ "Gesamtwirkung" gewollt sein. Verzicht und Vergleich gelten dann für alle Gesamtschuldner (ein Vergleich freilich nur, soweit er begünstigt). Darüber hinaus kann „Einzelwirkung “ dergestalt vereinbart sein, dass der Gläubiger lediglich darauf verzichtet, auf den Gesamtschuldner zuzugreifen, mit dem er sich geeinigt hat, nicht jedoch auf die Inanspruchnahme der übrigen Gesamtschuldner. Deren Haftung bleibt damit von einem Verzicht und Vergleich unbeeinflusst, was allerdings zur Konsequenz hat, dass sich der Erlasspartner weiterhin dem ungeschmälerten Gesamtschuldnerregress ausgesetzt sieht. ${ }^{48}$ Um dies zu vermeiden, kann der Parteiwille schließlich auf eine „beschränkte Gesamtwirkung “ gerichtet sein. Dabei wirken Verzicht und Vergleich gegenüber dem einzelnen Gesamtschuldner insoweit auch zu Gunsten der Mitschuldner, als der Gesamtschuldnerregress die Freistellung des Vertragspartners konterkarieren würde. ${ }^{49}$ Die übrigen Gesamtschuldner werden also - wie bei der Auflösung der „gestörten Gesamtschuld“ zu Lasten des Gläubigers von der auf den privilegierten Gesamtschuldner entfallenden Ausgleichsquote entbunden.

Bydlinski, aaO (Fn. 7), $\$ 423$ BGB Rdn. 3; StÜrner, aaO (Fn. 7), $\$ \int 422-424$ BGB Rdn. 1.

45 Dazu oben II.3.a).

46 Böttcher, aaO (Fn. 7), $\ 423$ BGB Rdn. 8; StÜrner, aaO (Fn. 7), $\mathbb{\int}$ 422-424 BGB Rdn. 5.

47 Bydlinski, aaO (Fn. 7), \423 BGB Rdn. 3; Gehrlein, aaO (Fn. 17), \423 BGB Rdn. 1.

48 Siehe etwa BGH NJW 2003, 2980, 2981.

49 BGH NJW 2012, 1071 Rdn. 22; BydLINski, aaO (Fn. 7), \423 BGB Rdn. 3. 


\section{b) Auslegungsregeln der bürgerlich-rechtlichen Dogmatik}

Nach der Rechtsprechung des BGH kommen Verzicht und Vergleich im Zweifel keine Gesamtwirkung zu. ${ }^{50}$ Auch soll es für die Annahme einer beschränkten Gesamtwirkung nicht per se genügen, dass der Vergleichspartner im Innenverhältnis den Schaden allein zu tragen hat; dies verbiete das grundsätzlich berechtigte Interesse des Gläubigers, sich bei den anderen Gesamtschuldnern schadlos halten zu können. ${ }^{51}$ Für den Willen, den Erlasspartner auch von dem Risiko zu befreien, dass der Vergleich durch einen Gesamtschuldnerausgleich ganz oder teilweise wertlos wird, müssten daher stets Anhaltspunkte im Vergleich oder den ihm zu Grunde liegenden Verhandlungen gefunden werden. ${ }^{52} \mathrm{Im}$ Schrifttum wird demgegenüber teilweise für die grundsätzliche Annahme beschränkter Gesamtwirkung plädiert, da ein Verzicht in der Regel darauf abziele, den Erlasspartner endgültig freizustellen, und auch ein Vergleich dahin zu Ende gedacht werden müsse, dass das Nachgeben des Gläubigers nicht durch den Gesamtschuldnerregress konterkariert werden dürfe; zudem lasse sich einem Vergleich ohnehin regelmäßig eine Entlastungsverpflichtung des Gläubigers im Ausmaß des Erlasses entnehmen, was dann zu einem Regresskreisel mit demselben wirtschaftlichen Ergebnis führe. ${ }^{53}$

\section{Beschränkte Gesamtwirkung im Rabmen der aktienrechtlichen Organhaftung?}

\section{a) Konsequenzen der Übertragung der bürgerlich-rechtlichen Dogmatik}

Überträgt man die vorstehenden Erwägungen auf die gesamtschuldnerische Haftung von Organmitgliedern gemäß $\$ 93$ Abs. 2 Satz 1 AktG, so ist zunächst zu konstatieren, dass eine beschränkte Gesamtwirkung von Verzicht und Vergleich jedenfalls durch eine unmissverständliche Formulierung, dass das Organmitglied endgültig im vereinbarten Umfang von der Haftung freigestellt werden soll, erreicht werden könnte. Angesichts der dreijährigen Sperrfrist des $₫ 93$ Abs. 4 Satz 3 AktG, welche gewährleisten soll, dass die Entscheidung über Verzicht und Vergleich erst zu einem Zeitpunkt getroffen

50 BGH NJW 2012, 1071 Rdn. 21; BGH NJW-RR 2005, 34, 36; BGH NJW 2000, 1942, 1943.

51 BGH NJW 2012, 1071 Rdn. 23; siehe aber auch BGH NJW 2000, 1942, 1943 („kann sich im Einzelfall daraus ergeben“).

52 BGH NJW 2012, 1071 Rdn. 23.

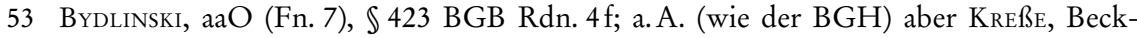

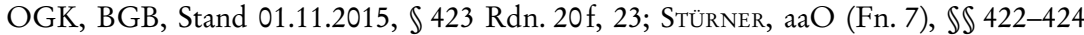
BGB Rdn. 3. 
wird, in dem sich das Ausmaß der Schädigung klar erkennen lässt, ${ }^{54}$ dürfte darüber hinaus sogar grundsätzlich anzunehmen sein, dass das Organmitglied auf die Endgültigkeit des Verzichts bzw. Vergleichs vertrauen können soll.

Indes führte die Annahme einer beschränkten Gesamtwirkung dazu, dass die übrigen Organmitglieder gleichsam en passant und ohne Einhaltung der Kautelen des $\ 93$ Abs. 4 Satz 3 AktG mit enthaftet würden. ${ }^{55}$ Das führte nicht zuletzt zu ganz erheblichen Problemen, wenn Vorstands- und Aufsichtsratsmitglieder wegen eines Überwachungsverschuldens des Aufsichtsrats als Gesamtschuldner haften: In diesem Fall müssen die für die Pflichtverletzung unmittelbar verantwortlichen Vorstandsmitglieder im Innenverhältnis den Schaden allein tragen. ${ }^{56}$ Wollte man einem Verzicht oder Vergleich mit diesen Vorstandsmitgliedern beschränkte Gesamtwirkung beimessen, würden die Mitglieder des Aufsichtsrates hierdurch vollständig enthaftet. Das würde freilich handfeste Interessenkonflikte zu Tage fördern, da der Aufsichtsrat nun versucht sein könnte, der Hauptversammlung einen weitestgehenden Erlass der Ansprüche gegen die Vorstandsmitglieder - sei es durch Verzicht oder Vergleich - vorzuschlagen, nur um einer eigenen Inanspruchnahme zu entgehen. ${ }^{57}$ Darüber hinaus täten sich unerwartete Kompetenzprobleme auf. Denn enthafteten Verzicht und Vergleich als Verfügungen zugunsten Dritter auch die Aufsichtsratsmitglieder, würde der Aufsichtsrat die Gesellschaft insoweit seinen eigenen Mitgliedern gegenüber vertreten. Daran änderte auch die Beteiligung der Vorstandsmitglieder am Vergleichsschluss nichts, da diese beim Vertragsschluss im eigenen Namen und nicht als Vertreter der Gesellschaft agieren.

\section{b) Unvereinbarkeit mit $\int 93 \mathrm{Abs} .4 \mathrm{Satz} 3 \mathrm{Akt} G$}

Letztlich scheitert die Annahme einer beschränkten wie unbeschränkten Gesamtwirkung eines zwischen Gesellschaft und einem einzelnen Organmitglied geschlossenen Verzichts oder Vergleichs jedoch bereits an $\ 93$ Abs. 4 Satz 3 AktG. ${ }^{58}$

54 Begr. RegE AktG 1965, abgedruckt bei KropfF, AktG, 1965, S. 123; Koch, aaO (Fn. 36), \} 9 3 \text { AktG Rdn. 76; Mertens/Cahn, aaO (Fn. 9), \93 AktG Rdn. } 1 6 4 .

55 Dies erkennend, indes ohne jegliches Problembewusstsein Schnorbus/Ganzer, WM 2015, 1877, 1885.

56 Dazu oben II.2.

57 In diesem Sinne das Ermessen des Aufsichtsrats beim Vergleich über Haftungsansprüche gegen Vorstandsmitglieder einschränkend Mertens/Cahn, aaO (Fn. 9), $\$ 93$ AktG Rdn. 163; dem folgend FleisCher, AG 2015, 133, 135; dagegen aber bereits BAYER/ Scholz, ZIP 2015, 149, $153 \mathrm{f}$.

58 Für die gesamtschuldnerische Haftung von Vorstands- und Aufsichtsratsmitgliedern bereits BAYER/SCHOLZ, ZIP 2015, 149, $153 \mathrm{f}$. 
Denn der Regelung ist ein konkludenter Verzicht unbekannt: Zum einen gelten Sperrfrist, das Erfordernis der Hauptversammlungszustimmung und das Minderheitenquorum für jeden einzelnen Anspruch, auf den verzichtet bzw. über den sich verglichen wird. Das ergibt sich bereits aus der Formulierung der Sperrfrist („nach der Entstehung des Anspruchs“) und wird durch den systematischen Zusammenhang zwischen Minderheitenquorum und Aktionärsklage $e^{59}$ bestätigt. Zum anderen genügt nach allgemeiner Auffassung für die erforderliche Zustimmung der Hauptversammlung eben nur ein formeller Hauptversammlungsbeschluss und die Sperrfrist soll gerade verhindern, dass die Hauptversammlung im Zeitpunkt der Beschlussfassung die Auswirkungen des Verzichts bzw. Vergleichs nicht überschauen kann. Verzicht und Vergleich gegenüber Organmitgliedern müssen nach der gesetzlichen Konzeption folglich als solche benannt der Hauptversammlung vorgelegt werden. Die Annahme einer beschränkten Gesamtwirkung führte demgegenüber dazu, dass alle übrigen mithaftenden Organmitglieder - sei ihre Haftung bekannt oder unbekannt - im Umfang der internen Haftungsquote des Vergleichspartners - sei diese bekannt oder unbekannt - enthaftet würden. Ein konkludenter Verzicht im Sinne einer beschränkten Gesamtwirkung des von der Hauptversammlung sanktionierten Verzichts oder Vergleichs genügt mithin nicht den Anforderungen des $₫ 93$ Abs. 4 Satz 3 AktG.

Dieses streng formale Verständnis von $\mathbb{} 93$ Abs. 4 Satz 3 AktG wird durch die Regelung des $₫ 124$ Abs. 2 Satz 3 AktG ( $\ 124$ Abs. 2 Satz 2 AktG a. F.) bestätigt. Danach ist der wesentliche Inhalt des Verzichts bzw. Vergleichs - also allen voran die Vertragsparteien und der konkrete Umfang des Anspruchsverzichts - im Vorfeld bekanntzumachen. Zwar könnte man auf den Gedanken kommen, eine beschränkte Gesamtwirkung gerade mit Blick auf $\$ 124$ Abs. 2 Satz 3 AktG anzunehmen, wenn in der Bekanntmachung explizit auf diese hingewiesen wird. Indes soll die Bekanntmachungspflicht den Aktionären lediglich eine angemessene Urteilsbildung ermöglichen. ${ }^{60}$ Sie ist nicht als Gestaltungsvariante erdacht, um in einem Vergleich oder Verzicht einen weiteren Verzicht zu verstecken. Vielmehr handelt es sich bei den in der beschränkten Gesamtwirkung liegenden (Teil-)Verzichten gegenüber den übrigen Gesamtschuldnern ihrerseits um Verträge, die nur mit Zustimmung der Hauptversammlung wirksam werden und für die die Bekanntmachungspflicht des $\ 124$ Abs. 2 Satz 3 AktG ebenfalls Geltung beansprucht.

Im Ergebnis bleibt damit festzuhalten, dass nicht einmal eine explizite Aufnahme der beschränkten Gesamtwirkung in den Vergleichsvertrag diese zu begründen vermochte, da die Voraussetzungen des $\ 93$ Abs. 4 Satz 3 AktG im Hinblick auf die darin liegenden (Teil-)Verzichte gegenüber den anderen Ge- 
samtschuldnern nicht erfüllt sind. Das heißt freilich nicht, dass es unmöglich wäre, einen Verzicht oder Vergleich gegenüber Organmitgliedern gegen dessen Aushöhlung durch den Gesamtschuldnerregress auszustatten. Nur bedürfen die hierfür erforderlichen Verzichte gegenüber den anderen Gesamtschuldnern einer expliziten Zustimmung der Hauptversammlung. Da es sich bei der „beschränkten Gesamtwirkung " nicht um ein gesetzliches Rechtsinstitut, sondern lediglich um einen im juristischen Diskurs gebildeten Begriff handelt, bricht ihr Ausschluss im Rahmen der aktienrechtlichen Organhaftung auch nicht mit der bürgerlich-rechtlichen Dogmatik.

Dass $\ 93$ Abs. 4 Satz 3 AktG für jeden Verzicht gilt, auch wenn er „nur“ einen anderen Verzicht oder Vergleich absichert, sollte kein Organmitglied überraschen.

\section{Zulässigkeit einer Freistellungszusage für Regressansprüche der übrigen Organmitglieder?}

Obwohl eine Absicherung gegen die Aushöhlung durch den Gesamtschuldnerregress somit nicht ausgeschlossen ist, lässt sich nicht in Abrede stellen, dass Verzicht und Vergleich über Organhaftungsansprüche ohne beschränkte Gesamtwirkung dem betroffenen Organmitglied nur bedingt weiterhelfen. Vergleicht sich der Aufsichtsrat nicht mit allen in den Haftungsfall involvierten Vorstandsmitgliedern, hat dies nämlich in aller Regel den Grund, dass ein entsprechender Vergleich mit den übrigen Vorständen nicht zu Stande gekommen ist. Es wäre der Hauptversammlung unter diesen Umständen nur äußerst schwer vermittelbar, dass ausgerechnet gegenüber den Vorstandsmitgliedern, mit denen sich der Aufsichtsrat nicht auf einen Vergleich einigen konnte, anteilig auf den Anspruch verzichtet werden sollte. Der Wunsch nach einer geräuschlosen Absicherung des Vergleichs gegen die Risiken aus dem Gesamtschuldnerregress erscheint daher durchaus nachvollziehbar. Allerdings zielt $\$ 93$ Abs. 4 Satz 3 AktG gerade darauf ab, eine gleichsam heimliche Enthaftung zu unterbinden. Nach den gesetzlichen Wertungen führt an sich kein Weg an einem expliziten und der Hauptversammlung vorzulegenden (anteiligen) Verzicht gegenüber den übrigen gesamtschuldnerisch haftenden Organmitgliedern vorbei.

\section{a) Unwirksamkeit einer pauschalen Freistellungspflicht für den Fall des Gesamtschuldnerregresses}

Aus diesem Grund begegnet es auch Bedenken, wenn im Schrifttum stattdessen empfohlen wird, eine Freistellungsverpflichtung der Gesellschaft für den Fall eines Gesamtschuldnerregresses durch nicht vergleichsbereite Vor- 
standsmitglieder in den Vergleichsvertrag aufzunehmen, ${ }^{61}$ wie es etwa bei der Aufarbeitung der Siemens-Korruptionsaffäre praktiziert wurde ${ }^{62}$. Eine solche Freistellungspflicht setzt nämlich einen Regresskreisel in Gang, bei dem das nicht befreite Organmitglied letzten Endes doch auf Kosten der Gesellschaft enthaftet wird, die Gesellschaft also de facto auch gegenüber diesem auf den Ersatzanspruch verzichtet. Auch wenn damit lediglich das durch Verzicht oder Vergleich ordnungsgemäß aus der Haftung entlassene Organmitglied abgesichert werden soll: Die Freistellungspflicht bewirkt - als notwendige Konsequenz - zielgerichtet die Enthaftung der nicht befreiten Organmitglieder.

Aus diesem Grund wäre es auch verfehlt, die Zulässigkeit einer solchen Freistellungszusage aus der Entscheidung des BGH zur Zulässigkeit der Übernahme einer Geldstrafe für Vorstandsmitglieder ${ }^{63}$ herleiten zu wollen. Zwar ging es dort auch um eine Freistellung von Organmitgliedern durch die Gesellschaft. Diese Freistellung hatte indes nicht die hier problematische Konsequenz einer mittelbaren Enthaftung weiterer Organmitglieder. Daher wäre auch mit dem Verweis auf das in selbiger Entscheidung anerkannte „Selbstschädigungsrecht" der Aktionäre nichts gewonnen. ${ }^{64}$ Denn selbstverständlich fällt auch ein Verzicht unter diese Kategorie und es steht auch außer Frage, dass diese Entscheidung in der Hand der Aktionäre liegen muss. Es geht vielmehr darum, dass der Gesetzgeber für den Verzicht auf Haftungsansprüche ein spezielles - formales und transparentes - Verfahren bereitgestellt und mit der Sanktion der Nichtigkeit im Falle der Nichtbefolgung bewehrt hat.

Daher ist jedenfalls eine pauschale Freistellungspflicht der Gesellschaft, bei der nicht erkennbar ist, welche anderen Organmitglieder in welchem Umfang enthaftet werden, als Umgehung des $\ 93$ Abs. 4 Satz 3 AktG zu werten. Sie ist somit ebenso unwirksam wie ein versteckter Verzicht gegenüber den übrigen Organmitgliedern durch Vereinbarung beschränkter Gesamtwirkung. ${ }^{65}$

Indes führt die Unwirksamkeit der Freistellungsklausel nicht gemäß $\$ 139$ BGB zur Gesamtnichtigkeit des Vertrages. Denn ein Verzicht oder Vergleich verliert durch den Wegfall des Freistellungsanspruchs nicht seine Funktion:

61 Seibt/Cziupka, DB 2014, 1598, These 20; zustimmend Hopt/Roth, aaO (Fn. 2), \ 93 AktG Rdn. 462.

62 Vgl. jeweils $\$ 3$ Abs. 1 lit. a) der Vergleichsverträge vom 02.12.2009 zwischen der Siemens AG und ihren (ehemaligen) Organmitgliedern sowie des Vergleichsvertrags zwischen der Siemens AG und Heinz-Joachim Neubürger (Einladungen zu den Hauptversammlungen der Siemens AG vom 26.01.2010, Anlagen zu TOP 12, und vom 27.01.2015, Anlage zu TOP 11).

63 BGHZ 202, 26.

64 BGHZ 202, 26 Rdn. 20.

65 Dazu oben III.2.b). 
Zum einen wird das Organmitglied gegenüber der Gesellschaft unmittelbar enthaftet. Zum anderen ermöglicht der Gesamtschuldnerausgleich den Regress nur im Umfang der auf dieses Organmitglied entfallenden Haftungsquote und entsprechende Ansprüche müssen überdies erst einmal (rechtzeitig) geltend gemacht werden.

b) Wirksamkeit von Freistellungsvereinbarungen für den Fall des Gesamtschuldnerregresses bei hinreichender Konkretisierung?

Es bleibt die Frage, ob eine Freistellungszusage der Gesellschaft für den Fall der Inanspruchnahme des ordnungsgemäß enthafteten Organmitglieds im Wege des Gesamtschuldnerregresses stets eine unzulässige Umgehung von \93 Abs. 4 Satz 3 AktG darstellt.

aa) Umgehung von $\int 93$ Abs. 4 Satz 3 Akt G auch bei Konkretisierung des Freistellungsumfangs und Eingrenzung auf bestimmte Regressfälle

Insofern erfordert der Normzweck des $₫ 93$ Abs. 4 Satz 3 AktG jedenfalls, dass für die Hauptversammlung im Zeitpunkt des Beschlusses erkennbar ist, in welchem Umfang und zugunsten welcher anderen Organmitglieder die Freistellung wirkt. Dementsprechend müsste eine im Vergleich oder Verzicht enthaltene Freistellungszusage für den Fall des Gesamtschuldnerregresses zumindest eine bezifferte Obergrenze enthalten und explizit auf den Regress durch bestimmte Vorstands- und Aufsichtsratsmitglieder beschränkt sein. Das allein vermag jedoch die Zulässigkeit einer Freistellungszusage noch nicht zu begründen, solange diese bei funktionaler Betrachtung nichts anderes als einen der Hauptversammlung gemäß $\$ 93$ Abs. 4 Satz 3 AktG separat vorzulegenden Teilverzicht gegenüber den übrigen Organmitgliedern bezweckt.

Freilich geht der Gesellschaft infolge der Freistellungszusage der Anspruch gegen die übrigen Gesamtschuldner nicht unmittelbar verloren. Es kann aber schwerlich einen Unterschied machen, dass ein in Anspruch genommener Gesamtschuldner auf einen Regress verzichten oder das in Regress genommene Organmitglied von der Durchsetzung des Freistellungsanspruchs gegen die Gesellschaft Abstand nehmen könnte. Wollte man sich darauf verlassen, dass Regressansprüchen nicht geltend gemacht werden, bedürfte es der Freistellungsvereinbarung nicht. In diesem Sinne macht es auch keinen Unterschied, dass sich die Gesellschaft der Inanspruchnahme aus der Freistellungszusage möglicherweise entziehen könnte, indem sie den Anspruch aus $\ 93$ Abs. 4 Satz 3 AktG gegenüber den übrigen Gesamtschuldnern unmittelbar vor Ablauf der Verjährungsfrist per Demnächstzustellung ( $\mathbb{1 6 7}$ ZPO) einklagt und so einen Gesamtschuldnerregress aus übergegangenem Recht gemäß $\ 426$ 
Abs. 2 BGB verhindert. ${ }^{66}$ Denn dieser Weg zum Schutz des ordnungsgemäß enthafteten Organmitglieds stünde der Gesellschaft - sofern er überhaupt zulässig ist $\mathrm{t}^{67}$ - auch ohne eine Freistellungsvereinbarung offen.

Mit der Vereinbarung von Freistellungszusagen für den Gesamtschuldnerregress ist somit kein Mehrwert verbunden, der es rechtfertigen könnte, diese nicht als Umgehung des gesetzlich vorgesehenen Procederes für eine Enthaftung von Organmitgliedern durch Verzicht und Vergleich gemäß $\$ 93$ Abs. 4 Satz 3 AktG zu begreifen.

\section{bb) Komplikationen mit $\int 93 \mathrm{Abs} .5 \mathrm{Akt} G$}

Die Zulassung von Freistellungsvereinbarungen für den Fall des Gesamtschuldnerregresses wäre schließlich nicht nur mit Blick auf $\$ 93$ Abs. 4 Satz 3 AktG, sondern auch im Hinblick auf die Regelung des $₫ 93$ Abs. 5 AktG mit Problemen verbunden.

Nach $₫ 93$ Abs. 5 Satz 3 AktG wird die Ersatzpflicht der Organmitglieder im Fall der Anspruchsdurchsetzung durch die Gesellschaftsgläubiger nach $\$ 93$ Abs. 5 Satz 1 AktG nämlich weder durch einen Verzicht noch einen Vergleich der Gesellschaft aufgehoben. Diese jedem Verzicht immanenten Grenzen darf eine Freistellungsvereinbarung freilich nicht unterlaufen. Doch wollte man $\$ 93$ Abs. 5 Satz 3 AktG per analoger Anwendung auf Freistellungsvereinbarungen erstrecken, hieße das erst einmal nur, dass auch eine Freistellungsvereinbarung den Gläubigern gegenüber die Ersatzpflicht aus $₫ 93 \mathrm{Abs} .2 \mathrm{AktG}$ nicht beseitigt. Das ist aber ohnehin der Fall, weil die Freistellung den Anspruch der Gesellschaft nicht direkt abschneidet. Um dem Normzweck des Gläubigerverfolgungsrechts gerecht zu werden, müsste man also einen Anspruch der Gesellschaft auf Rückzahlung bereits geleisteter Freistellungszahlungen entsprechend $\ 812$ Abs. 1 Satz 2 Var. 1 BGB (nachträglicher Wegfall des rechtlichen Grundes) begründen und für diesen wiederum die Durchsetzung durch die Gesellschaftsgläubiger anerkennen.

Indes ist das Verfolgungsrecht der Gläubiger - und damit auch die Unwirksamkeitsfolge des $\ 93$ Abs. 5 Satz 3 AktG - gemäß $\$ 93$ Abs. 5 Satz 2 AktG

67 Im Schrifttum wird zum Teil sogar erwogen, dass die Treupflicht es gebiete, Organmitglieder bei besonders hohen Schäden und vergleichsweise geringen Verursachungsbeiträgen nur nach ihrer internen Haftungsquote in Anspruch zu nehmen (so MerTeNs/ CAHn, aaO (Fn. 9), $\$ 93 \mathrm{AktG}$ Rdn. 53). Indes besteht für die Gesamtschuldner die Möglichkeit, ihren Ausgleichsanspruch aus $₫ 426$ Abs. 1 BGB abzusichern (dazu FISCHER, ZIP 2014, 406, 408ff). Insofern vermag eine kurz vor Ablauf der Verjährung des Anspruchs aus $\ 93$ Abs. 2 AktG erhobene Klage das in Anspruch genommene Organmitglied nicht völlig schutzlos zu stellen. 
auf Fälle grober Pflichtverletzungen der Organmitglieder beschränkt. Das macht die Situation noch komplizierter. Denn einerseits leistet die Gesellschaft stets auf Grund der im Vergleich getroffenen Freistellungsvereinbarung, auch wenn sie nicht an das ordnungsgemäß enthaftete Organmitglied zahlt, nachdem dieses in Regress genommen wurde, sondern direkt an das Organmitglied, das den Regressanspruch geltend macht. Schuldner des Rückgewähranspruchs wäre daher nach allgemeinen Grundsätzen stets das ordnungsgemäß enthaftete Organmitglied, zumal $\$ 93$ Abs. 5 AktG nach seinem Sinn und Zweck das Innenverhältnis der gesamtschuldnerisch haftenden Organmitglieder nicht tangiert. Auf der anderen Seite verlangt $\mathbb{9} 93$ Abs. 5 Satz 2 AktG, den Blick auf die Pflichtverletzungen der auf Grund der Freistellung faktisch mitenthafteten Organmitglieder zu richten. In der Konsequenz müssten daher Freistellungszahlungen von dem ordnungsgemäß enthafteten Organmitglied ungeachtet der Schwere der diesem zur Last fallenden Pflichtverletzung kondiziert werden können, sofern sie nur im Hinblick auf den Gesamtschuldnerregress durch ein Organmitglied erfolgten, dem eine grobe Pflichtverletzung vorgeworfen werden kann. Darüber hinaus stellte sich sogar die Frage, ob das freigestellte Organmitglied auch und bereits zur Rückgewähr verpflichtet wäre, wenn ihm selbst eine grobe Pflichtverletzung vorgeworfen werden könnte. Denn unter diesen Voraussetzungen stünde der Vergleich gemäß $₫ 93$ Abs. 5 Satz 3 AktG der Anspruchsverfolgung durch die Gesellschaftsgläubiger nicht entgegen, was dann wohl auch für die in diesem Vergleich vereinbarte Freistellungspflicht gelten müsste.

Freilich ließen sich all diese Probleme unter Rekurs auf allgemeine bereicherungsrechtliche Grundsätze und die $\ 93$ Abs. 5 AktG zu Grunde liegenden gesetzgeberischen Wertungen auflösen. Dass es allein mit einer analogen Anwendung des $₫ 93$ Abs. 5 Satz 3 AktG nicht getan wäre, untermauert jedoch eher die Einschätzung, dass im System der aktienrechtlichen Organhaftung kein Raum für eine Freistellung von Organmitgliedern für den Fall des Gesamtschuldnerregresses ist.

Im Ergebnis vermag daher auch eine Konkretisierung der Freistellungsklausel nichts an der Qualifikation als unzulässige Umgehung eines der Hauptversammlung separat vorzulegenden Teilverzichts gegenüber den übrigen gesamtschuldnerisch haftenden Organmitgliedern zu ändern.

\section{Konsequenz: Beschränkte Gesamtwirkung als rechtspolitisches Desiderat?}

$\mathrm{Da}$ es nach der lex lata weder möglich ist, einem nach $\$ 93$ Abs. 4 Satz 3 AktG von der Hauptversammlung gebilligten Verzicht oder Vergleich beschränkte Gesamtwirkung beizumessen noch diesen mit einer Freistellungszusage für den Fall des Gesamtschuldnerregresses auszustatten, drängt sich die Frage auf, ob insofern rechtspolitischer Handlungsbedarf besteht. Dabei dürfte nach den 
vorstehenden Überlegungen auf der Hand liegen, dass jedenfalls keine Notwendigkeit für die Zulassung von Freistellungszusagen besteht. Anders liegt die Sache jedoch bei der beschränkten Gesamtwirkung. Denn es ist in der Tat unbefriedigend, wenn die Enthaftung eines Organmitglieds trotz Einhaltung des gesetzlich vorgeschriebenen Procederes aus Abwarten der Dreijahresfrist und Hauptversammlungsbeschluss letztlich doch keine endgültige Wirkung zeitigt. Die de lege lata bestehenden Bedenken sind jedoch auch in der rechtspolitischen Perspektive nicht von der Hand zu weisen: Die äußerst strengen und auf Transparenz ausgelegten Anforderungen an die Wirksamkeit von Verzicht und Vergleich gehen nur schwer mit der unbestimmten Enthaftung einer unbestimmten Anzahl weiterer Organmitglieder zusammen. Eine beschränkte Gesamtwirkung sollte daher de lege ferenda nur unter der Voraussetzung anerkannt werden, dass der der Hauptversammlung vorgelegte Verzicht bzw. Vergleich (i) die Gesamtwirkung als solche bezeichnet, (ii) den Umfang des gegenüber den anderen Gesamtschuldnern erklärten Teilverzichts konkret beziffert und (iii) die drittbegünstigten Organmitglieder namentlich benennt.

\section{Fazit}

$\mathrm{Ob}$ das arbeitsrechtliche Haftungsprivileg im Falle der gesamtschuldnerischen Haftung von Organmitgliedern und Arbeitnehmern mittelbar zur Enthaftung der Erstgenannten führt, ist eine ebenso berechtigte Frage wie jene nach den Auswirkungen von Verzicht und Vergleich gegenüber einem Organmitglied auf die Haftung der mitverantwortlichen Organmitglieder. Denn nach herrschender Auffassung im bürgerlich-rechtlichen Schrifttum wird die „gestörte Gesamtschuld“ zwischen Arbeitnehmern und Dritten zu Lasten des Arbeitgebers aufgelöst und einem Verzicht kann, ebenso wie einem Vergleich, durchaus „beschränkte Gesamtwirkung“ zukommen. Schließlich droht die - anfängliche bzw. nachträgliche - Haftungsprivilegierung in beiden Fällen durch den Gesamtschuldnerausgleich konterkariert zu werden. Die vorstehenden Überlegungen haben indes gezeigt, dass beide Fragen zu verneinen sind: $\ 93$ Abs. 4 Satz 3 AktG steht sowohl einer Auflösung der „gestörten Gesamtschuld“ zu Lasten der Gesellschaft als auch einem konkludent miterklärten Verzicht gegenüber den übrigen Organmitgliedern entgegen. Das gilt ebenso für Freistellungszusagen, die auf den Fall der Inanspruchnahme im Wege des Gesamtschuldnerregresses abheben. Rechtspolitisch erscheint die gesetzliche Anerkennung der beschränkten Gesamtwirkung von Vergleichen allerdings geboten. Insofern zeigt sich einmal mehr der Reformbedarf der aktienrechtlichen Organhaftung. ${ }^{68}$

68 Für weitreichende Reformen zuletzt die Abteilung Wirtschaftsrecht des 70. DJT vom 18.09.2014 (Beschlüsse abgedruckt in ZIP 2014, 1902) auf Grundlage von BAchmann, 
Bis zum Einschreiten des Gesetzgebers ist eine Haftungsbegrenzung ohne Rechtsfortbildung ${ }^{69}$ oder expliziten Hauptversammlungsbeschluss ${ }^{70}$ jedoch auch in den Untiefen der Gesamtschulddogmatik nicht zu finden.

Gutachten E für den 70. Deutschen Juristentag, 2014, S. 28 ff; dazu im Vorfeld BAYER, NJW 2014, 2546; BAYER/SchOlz, NZG 2014, 926; ausführlich zur Notwendigkeit einer Reform der Vorstandshaftung Scholz, aaO (Fn. 1), S. $227 \mathrm{ff}$.

$69 \mathrm{Zu}$ den de lege lata bestehenden Möglichkeiten ausführlich Scholz, aaO (Fn. 1), S. $265 \mathrm{ff}$; konzis BAYER/SCHOLZ, GmbHR 2015, 449, 455f; aktueller Meinungsstand bei Косн, aаO (Fn. 36), \93 AktG Rdn. 51f; siehe neuerdings auch Binder, Grenzen der Vorstandshaftung, 2016, S. $271 \mathrm{ff}$; BROMmER, Die Beschränkung der Rechtsfolgen der Vorstandsinnenhaftung, 2016, S. $209 \mathrm{ff}$.

$70 \mathrm{Zu}$ den Pflichten von Aufsichtsrat und Hauptversammlung bei Verzicht und Vergleich über Haftungsansprüche gegen Vorstandsmitglieder eingehend BAYER/SCHOLZ, ZIP $2015,149$. 